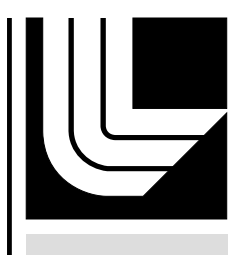

LAW RENCE LIVERMORE N A T IO N A L LABORATORY

\title{
Using Plasma Physics to Weigh the Photon
}

D.D. Ryutov

June 28, 2007

Plasma Physics and Controlled Fusion 
This document was prepared as an account of work sponsored by an agency of the United States Government. Neither the United States Government nor the University of California nor any of their employees, makes any warranty, express or implied, or assumes any legal liability or responsibility for the accuracy, completeness, or usefulness of any information, apparatus, product, or process disclosed, or represents that its use would not infringe privately owned rights. Reference herein to any specific commercial product, process, or service by trade name, trademark, manufacturer, or otherwise, does not necessarily constitute or imply its endorsement, recommendation, or favoring by the United States Government or the University of California. The views and opinions of authors expressed herein do not necessarily state or reflect those of the United States Government or the University of California, and shall not be used for advertising or product endorsement purposes. 


\title{
Using Plasma Physics to Weigh the Photon
}

\author{
D.D. Ryutov \\ Lawrence Livermore National Laboratory, Livermore, CA 94551, USA
}

\begin{abstract}
The currently accepted value for the upper bound for the photon mass $m_{p h}$ is 22 orders of magnitude less than the electron mass. As the mass $m_{p h}$ is so incredibly small, it has essentially no effect on the atomic and nuclear physics, and it is very difficult to improve this estimate by laboratory experiments. However, even a very small mass may have significant effect on astrophysical phenomena occurring at the scale exceeding the photon Compton length $\lambda$ (where $\lambda \sim 3$ million kilometers for the currently accepted mass). Based on the set of MHD equations (written with the account for the finite photon mass), the author analyzes properties of the Solar wind at the Pluto orbit and comes up with an improved (reduced) by a factor of 70 estimate of the photon mass. Possible opportunities and challenges for the further reduction of the upper limit for $m_{p h}$ based on the properties of larger-scale astrophysical objects, are discussed.
\end{abstract}

\section{Introduction}

Contrary to sometimes professed views, the finiteness of the photon mass is compatible with the Einstein relativity theory. The quantum description of the finite-mass spin-one particle was developed by Proca [1]; for a modern exposition see, e.g., Landau and Lifshitz [2]. The appropriately modified Maxwell equations (the classical limit of the Proca equations) are presented in a number of textbooks, including famous Jackson's "Classical Electrodynamics" [3]. General discussion of the earlier work on the massive photon has been presented in an excellent review paper [4]; later developments have been discussed in reviews [5-9].

The currently accepted value for the upper bound of the photon mass [10] is impressively low,

$m_{p h}<10^{-22} m_{e} \approx 10^{-51} \mathrm{~g}$,

where $m_{e}$ is the electron mass. Certainly, so low a photon mass would not have any impact on, say, atomic physics. As the mass (even if finite), is incredibly small, it is very difficult to improve estimate (1) by laboratory experiments (see discussion in [11, 12]).

One of the consequences of the finiteness of the photon mass is that the magnetic field created by a magnetic dipole would decrease not in a dipole fashion, $\sim 1 / r^{3}$, but rather in a Yukawa-like fashion, $\exp (-r / \hbar) / r^{3}$, where $\lambda$ is the photon Compton length, $\lambda=\hbar / m_{p h} c$;

here $\hbar$ is the Planck constant and $c$ is the speed of light. [Strictly speaking, for the finite photon mass, the speed of light depends on the wavelength, as was first noticed by Louis DeBroglie [13]. It would be more precise to say that $c$ is the speed of light for the veryshort-wavelength photons.]. For the mass (1) the Compton length is $\sim 3 \times 10^{11} \mathrm{~cm}$. 
E. Schroedinger, who was the first to notice the appearance of the exponential cut-off in the dipole field, suggested using this effect for the evaluation of $\lambda$ (and $m_{p h}$ ) based on the measuring Earth's magnetic field [14]; his estimates ranged between $6 \times 10^{8}$ and $3 \times 10^{9} \mathrm{~cm}$. Clearly, the stronger the magnet, the further one can see its magnetic field, the better estimate for $\lambda$ one can make. In 1975 measurements of the Jupiter magnetic field by the Pioneer-10 spacecraft allowed pushing the limit to 0.3 million kilometers [15]. At larger distances, the Jovian field gets too strongly distorted by the Solar wind and MHD effects become important.

At this point, the potential importance of the plasma physics becomes clear: If one wants to study astrophysical objects with large-scale magnetic fields present, one has always to deal with a question of whether these fields are produced by some distant source (say, a dipole), or they are produced in situ, by the currents flowing in the local plasma. And, for large-enough distances one would always deal with the fields generated locally. This is why magnetohydrodynamic (MHD) effects have been recognized since 1960s as playing important role in the attempts to evaluate the photon mass based on astrophysical observations (see discussion of this issue in Ref. [4]).

Somewhat surprisingly, a complete set of MHD equations for the case of a finite photon mass has not been written until recently. It has been first derived and discussed in the author's paper [16]. Using this set and addressing the problem of the Solar wind sector structure at the Earth orbit, the author came up with the currently accepted estimate (1). One can note that the Compton length corresponding to this mass is 50 times shorter than the Earth-Sun distance (1 Astronomical Unit, AU). This is manifestation of the role of the ambient plasma: although the magnetic field of the Solar wind is certainly "born" on the Sun, it is "sustained" by the local plasma currents. This issue is illustrated in a more quantitative fashion below, in Sec. 3.1, where we also illustrate a "reductio ad absurdum" approach used to establish a lower bound on $\lambda$.

In this paper, we use information about the properties of the Solar wind at large distances from the Sun, at the orbit of Pluto, to improve our earlier estimate (1) by at least a factor of 70 (sec. 3.2). But, before that, in Sec. 2, for reference purpose, we present the set of MHD eqations of Ref. [16] and discuss their most salient features. After that, in Sec. 4, we consider the possibility of improving the mass estimate by looking of more distant astrophysical objects. Finally, in Sec. 5, we discuss main results of this paper.

It should be noted that all the discussion in this paper, as well as in the papers mentioned above is based on the Proca model of a massive photon. Should this way of description fail for some reason, our basic equations would also fail. In particular, if the Higgs boson model of the photon suggested in Ref. [17] is valid, our approach would remain valid only in one of the regimes discussed in Ref. [17].

One more comment is in order here. Getting extraterrestrial (in the terminology of Ref. [4]) limits on the photon mass is facilitated by large spatial scales involved. This allows one, even by a relatively crude analysis, to reach substantial progress. On the other hand, the terrestrial measurements can provide much higher accuracy, and probe relatively minor deviations from standard Maxwell equations. This approach may also yield interesting results: in a recent paper [18], by studying the propagation of ultra-low frequency waves in the space between the conducting Earth and the ionosphere, the author managed to bring the upper bound for $m_{p h}$ to $4 \times 10^{-52} \mathrm{~g}$, i.e., somewhat below the currently accepted value (1). 


\section{Magnetohydrodynamics with the finite photon mass}

The set of MHD equations for quasi-static processes (i.e., for the processes where both the fluid velocity and the wave velocity is much less than $c$ ) was first written in Ref. [16]. It reads as:

$$
\begin{aligned}
& \frac{\partial \rho}{\partial t}+\nabla(\rho \mathbf{v})=0 \\
& \rho \frac{d \mathbf{v}}{d t}=-\nabla p+\frac{1}{c} \boldsymbol{j} \times \boldsymbol{B} \\
& \nabla \times \nabla \times \boldsymbol{B}+\frac{\boldsymbol{B}}{\lambda^{2}}=\frac{4 \pi}{c} \nabla \times \boldsymbol{j} \\
& \nabla \times \boldsymbol{E}=-\frac{1}{c} \frac{\partial \boldsymbol{B}}{\partial t} \\
& \boldsymbol{j}=\sigma\left(E+\frac{\mathbf{v} \times \boldsymbol{B}}{c}\right)
\end{aligned}
$$

We use CGS system of units, with $\rho, p$, and $\mathbf{v}$ being the density, pressure and velocity of the fluid, $\boldsymbol{j}$ being the current density, and $\sigma$ being the electrical conductivity. The standard entropy equation can be added, if needed. As we have already mentioned, the electromagnetic retardation effects are ignored.

The set (3)-(7) coincides with the standard set of MHD equations in everything except for the second term in the left-hand-side (LHS) of Eq. (5), which accounts for the finiteness of the photon mass (we prefer to work with the equivalent characteristic, the photon Compton length $\lambda$ ). The key point in coming up with this set of equations is the realization that Eqs. (3), (4) and (7), are not changed by the finiteness of the photon mass. Indeed, one could derive them directly in the standard way, from the kinetic equations with particle collisions accounted for, and with no effect of the finite photon mass showing up - just because the mass is extremely small. One can apply the same arguments also to higher levels of the plasma description, based on kinetic equations. Obviously, two-fluid Braginski-type equations would also work. We, however, restrict ourselves to the single-fluid MHD.

A more formal way of looking at the problem is that Eqs. (3)-(7) are a direct corollary to the Proca Lagrangian, as presented, e.g., in Ref. [3].

Some general properties of the set (3)-(7) have been discussed in Ref. [16]. The most important one is that, within the Proca MHD, there does not exist such an entity as a uniform magnetic field immersed in a uniform resting plasma. Indeed, the uniform field, according to Eq. (5), means the presence of the non-uniform current, not collinear to the uniform field. Then, as Eq. (4) shows, an equilibrium state of a uniform plasma becomes impossible. This simple observation shows that the simple solutions of MHD equations of the type of linear MHD waves in a uniform system become impossible (senseless). The waves would exist on the background of more-or-less complex non-uniform equilibrium states, some of which have been considered in Ref. [16]. 


\section{Solar wind with the finite photon mass.}

\subsection{Inside the Earth orbit}

One of the great achievements of the space physics is a good level of understanding of the average properties of the Solar wind [19, 20]. The results for the average flow follow reasonably well the model of a radially expanding plasma, that stretches the magnetic field present in the vicinity of the Sun. A dipole component of the field, when pulled by the expanding plasma, causes the appearance of the almost radially directed magnetic field, with the fluxtubes carrying the magnetic flux determined by the magnetic field in the vicinity of the Sun [21]. So, it may be tempting to say that, as we see significant magnetic field in the Solar wind at the Earth orbit, the exponential factor in the Yukawa-like solution should not be too small, meaning that the photon Compton length is greater than, say, $0.1 \mathrm{AU}$.

To demonstrate that this approach is misleading, we consider in some detail a purely kinematic model, where the plasma flow has only radial component, starting from some spherical surface, over which the distribution of the normal component of the magnetic field is given. To be specific, consider a dipole distribution,

$B_{r}=B_{0} \cos \theta$,

where $B_{0}$ is some normalization field, and the angle $\theta$ is a polar angle measured from the south pole; at the equator, $\theta=\pi / 2$, the normal component changes sign. The condition $\nabla \cdot \boldsymbol{B}=0$ then yields

$B_{r}=\frac{B_{0} r_{0}^{2} \cos \theta}{r^{2}}$.

This part of the problem is described by Eqs. (5) and (7), which are the same as in the standard MHD (we consider the case of perfect conductivity, which is adequate for the parameters of the Solar wind). Accordingly, our arguments here do not differ from those presented by Parker in his seminal paper [21].

From the symmetry of the problem, it is clear that the plasma current has only azimuthal $(\varphi)$ component, which can be found from equation (5):

$j_{\varphi}=\frac{c B_{0} r_{0}^{2}}{4 \pi r^{3}}\left(1-\frac{r^{2}}{\hbar^{2}}\right) \sin \theta$

The magnetic field and the current distribution are illustrated in Fig. 1. Note that there is no exponential factor either in the magnetic field or in the current density. This is due to the fact that the field (9) is generated by the local currents, not by the currents in the vicinity of the Sun. The solar currents generate local magnetic field and then this field is stretched by the plasma flow due to the line-tying constraint (7). The currents adjust themselves so as to produce the resulting magnetic field (9).

It is in the expression for the current where the finiteness of the photon mass shows up: see the last term in Eq. (10). At distances exceeding $\lambda$, the current changes sign and becomes much larger than it were in the absence of the finite photon mass effect. Unfortunately, the plasma current density is a quantity that is difficult to measure directly. Where the effect of the finite photon mass shows up in the form allowing for a direct comparison with observation, is the momentum equation.

The $j \times B$ force acts in the $\theta$ direction: 
$f_{\theta}=\frac{j_{\varphi} B_{r}}{c}=\frac{B_{0}^{2} r_{0}^{2} \sin \theta \cos \theta}{4 \pi r^{5}}\left(1-\frac{r^{2}}{\hbar^{2}}\right)$

For a zero photon mass, this force pushes plasma towards the (Solar) equatorial plane (i.e., to the south at the northern side, and to the north at the southern side). However, this force is quite small. On the other hand, if the photon mass is large enough, so that $\lambda$ is less than the radius $r$, the force changes sign and increases significantly. This force would push plasma to the poles, depleting the equatorial region. By "cranking up" the photon mass, one finally reaches a situation where the presence of this force becomes manifestly incompatible with the observed flow pattern. This signals that the mass is actually below the assumed value. This approach, used, in particular, in Ref. [16], was a basis for the estimate (1). In the following section, we apply it to the Solar wind at large distances from the Sun, at the Pluto orbit.

\subsection{At the outer edge of the heliosphere}

As has already been mentioned, the Solar rotation, in combination with the linetying, causes the magnetic field lines to be wound into the spiral, as was first suggested by E. Parker [21]. At the distances beyond the Mars orbit, the azimuthal $\left(B_{\varphi}\right)$ component of the magnetic field becomes dominant. At the orbit of Pluto, the radial component of the field is already less than $3 \%$ of the azimuthal component. As $B_{\varphi}$ is produced by the winding of the radial magnetic field, which has opposite polarity in the Northern and Southern hemispheres, $B_{\varphi}$ changes sign near the equatorial plane.

Her we again use the "reductio ad absurdum" approach. Assuming that the Compton length is significantly less than $40 \mathrm{AU}$, one would find that the current density, according to Eq. (5), has $r$ and $\theta$ components, of the same order of magnitude,

$j \sim \frac{c B_{\varphi} r}{4 \pi \hbar^{2}}$

The direction of the current is such as to cause the plasma expansion from the equator to the poles, and to slow down the radial expansion (Fig.2).

The $j \times B$ force (per unit volume) can be evaluated as

$$
f \sim \frac{B_{\varphi}^{2} r}{4 \pi \hbar^{2}}
$$

The presence of this force would cause deviation from the essentially radial expansion with the constant radial velocity $\mathrm{v}$ if the condition $f>\rho \mathrm{v}^{2} / r$ was satisfied. On the other hand, direct observations show that the solar wind velocity is largely radial, including a distant zone at the Pluto orbit, and the velocity is the same as at the Earth orbit, $450 \mathrm{~km} / \mathrm{s}$. This means that actually the inequality

$$
f<\frac{\rho \mathrm{v}^{2}}{r}
$$

holds, which limits $\lambda$ from below:

$$
\frac{\hbar}{r}>\frac{1}{q} \sqrt{\frac{B_{\varphi}^{2}}{4 \pi \rho \mathrm{v}^{2}}},
$$

where $q$ is a safety factor, accounting for the crudeness of our analysis. Taking a generous value of $q=3$, and $r=40 \mathrm{AU}$ (the Pluto orbit), and using the observational values 
of particle density, magnetic field, and Solar wind velocity at the Pluto orbit $\left(n=10^{-2} \mathrm{~cm}^{-3}\right.$, $\mathrm{v}=4.5 \times 10^{7} \mathrm{~cm} / \mathrm{s}, \mathrm{B}=2 \times 10^{-6} \mathrm{G}$, Ref. [22]), we find the following bound on the photon Compton length and photon mass:

$$
\lambda>2 \times 10^{13} \mathrm{~cm} ; \quad m_{p h}<1.5 \times 10^{-24} m_{e}=1.5 \times 10^{-53} \mathrm{~g} .
$$

This is a suggested new estimate of the photon Compton length and photon mass. It is improved by almost two orders of magnitude compared to the currenly accepted value (1).

This limit is based on direct in situ measurements and in this regard is as reliable as the limits established in ground-based laboratory experiments, especially given a large safety factor that we have used. No doubt that it can be improved by at least a factor of a few by more detailed numerical simulations of the flow pattern that would be produced by the set of equations (3)-(7) in the case where the mass would be further reduced compared to Eq. (16).

The current density in the case of small Compton length is much higher than it would have been in a "standard" MHD (see Eq. (12)). One might try to use this circumstance to obtain one more constraint on $\lambda$. (Using an evaluation of the current density for constraining $\lambda$ was suggested in Ref. [23].) The relative ("current") velocity of electrons and ions is:

$$
u=\frac{j}{e n}
$$

In our case, taking $\lambda$ as in Eq. (16), and the other parameters of the Solar wind at the Pluto orbit from Ref. [22]: we find that the ratio of to the electron thermal velocity is still very low, $u / \mathrm{v}_{T e}<10^{-5}$, this meaning that the effects of the anomalous resistivity would not play any significant role. One can check that the Joule dissipation is also small, and the line-tying condition holds well.

\section{Using other astrophysical objects for establishing $m_{p h}$}

\subsection{Galactic magnetic field}

A natural way to improve the estimate of the photon Compton length is to consider electromagnetic phenomena with as large spatial scales as possible. This has been recognized as early as in 1950s, when Yamaguchi [24] (referring to discussions with Thirring) suggested using the presence of the large-scale magnetic fields of astrophysical objects for this purpose. Specifically, he used the inferred presence of the magnetic fields in the Crab Nebula taking as the length scale. Goldhaber and Nieto [4] pointed out that the presence of the ambient conducting medium does not allow identifying the spatial scale with the Yukawa fall-off factor (p. 293 in Ref. [4]); they mentioned that some kind of energy balance consideration should rather be given. Chibisov [25] used a force balance discussion for the case of a Great Magellanic Cloud and, assuming that the scale of the magnetic field is of order of the size of the cloud, $1 \mathrm{kpc}$, concluded that the photon Compton length should exceed $1 \mathrm{kpc}$. More recently, [17], Chibisov's arguments were reproduced for our Galaxy, with reference to the magnetic field in the spiral arm of $\sim 1$ $\mu \mathrm{G}$ and the average pressure of the interstellar medium being of order of the magnetic 
pressure. Then such force balance considerations (cast in the form of a virial theorem) lead the authors of [17] to the conclusion that $\lambda>1 \mathrm{kpc}$. However, the authors of the biannual compendium "Review of particle physics" $[26,10]$ have consistently rejected estimate [25], referring to inapplicablility of the virial arguments in the problem under consideration. Indeed, the fact that the interstellar medium is not an isolated system, but is strongly gravitationally coupled to the stellar matter, and is affected by the processes like supernovae explosions, makes the use of the virial theorem somewhat difficult [27]. It has also to be remembered that, although a large amount of observational data has been collected and the presence of the large-scale Galactic magnetic field seems to be established, its magnitude, spatial structure, and the level of smaller-scale fluctuations are still an area of active research (see reviews [28, 29]). The most common technique for evaluating the global field, is detection of the Faraday rotation of electromagnetic radiation from pulsars. But this effect provides an information only regarding the lineintegrated product of the magnetic field and electron density. Therefore, its interpretation requires additional hypotheses; in particular, the correlation between the field strength and plasma density may lead to substantial bias in the interpretation of the measurements of the Faraday rotation [30].

\subsection{Magnetic field in galactic clusters}

One can hope that the improvement in our understanding of the structure of the interstellar magnetic field, based on the analysis of the constantly appearing new data, will lead to resolving of the uncertainties mentioned in the previous section. Then, just due to the large scale involved, significant improvements of the mass limit will become possible. In this regard, one should remember also of the presence of even larger structures, where the magnetic field plays significant role in the plasma dynamics, namely, the clusters of Galaxies [31].

The scale of typical clusters is in the range of $10 \mathrm{Mpc}$, and the predicted scale of the regions of a coherent magnetic field extends to $L \sim 0.5 \mathrm{Mpc}[32,33]$. Making an assumption regarding the energy equipartition between the plasma and the magnetic field, one finds, in the same way as in Refs. $[25,17]$ that strong deviations from the present models would appear if the photon Compton length was shorter than the characteristic scale $L$. Introducing safety factor $q \sim 5$ (see Sec. 3.2), one finds the following upper bound on $\lambda$ : $\lambda>L / 5 \sim 100 \mathrm{kpc}$. This conclusion, however, would be the subject of the same concerns as an estimate based on the assumed properties of the Galactic magnetic field (Sec. 4.1).

\subsection{Smaller-scale astrophysical objects}

In near term, there may be a better chance to improve the mass estimate by considering astrophysical objects of an intermediate scale (up to a few parsecs), like dense molecular clouds or stellar outflows. For the first group of objects there exist a large data base for the magnetic field strengths and/or geometrical structure [34]. There is also a growing understanding of the underlying dynamics, including the role of the gravitation and star formation (e.g., [35]) and of the magnetic field (e.g., [36], [37]). As 
these objects are numerous, well defined spatially, and well characterized, one can expect obtaining a reliable estimate of $m_{p h}$.

Protostellar and stellar outflows are also quite numerous, and a number of them are spatially well-resolved. The problem is that there are essentially no direct magnetic field measurements for such objects, although it is generally assumed that the magnetic field plays a significant role in their formation and collimation [38]. One can expect to obtain constraints on the photon mass from analyzing the equilibrium and stability properties of the pinch equilibria, as was suggested in Ref. [39]. Interestingly, there exists laboratory Z-pinch experiments [40] that produce structures strikingly similar to those observed on the sky, thereby supporting assumption regarding significant role of magnetic field in the stellar outflows.

\section{Discussion}

There exists a reliable theory framework for assessing MHD processes in the case where the finiteness of the photon mass is important. In the quasistatic limit (wave velocity and fluid velocity slow compared to the speed of light), the modification occurs in the Ampere law and is essential for MHD processes with the spatial scales greater than the photon Compton length $\lambda$, Eq. (1). In order to make the effect of such a large-scale field on the dynamics of the system important, the field should be strong-enough. So, when one wants to evaluate the photon mass based on the observations of a certain system, one has to have information about both the magnetic field and plasma.

Table 1. Some of the available estimates of the photon mass

\begin{tabular}{|l|l|l|l|l|l|l|l|}
\hline Reference & {$[15]$} & {$[16]$} & {$[18]$} & $\begin{array}{c}\text { This } \\
\text { paper }\end{array}$ & {$[10,17]$} & $\begin{array}{c}\text { This } \\
\text { paper }\end{array}$ & {$[4,10]$} \\
\hline Object & Jupiter & $\begin{array}{l}\text { Solar } \\
\text { wind at } 1 \\
\text { AU }\end{array}$ & $\begin{array}{l}\text { Ultra-low } \\
\text { frequency } \\
\text { waves }\end{array}$ & $\begin{array}{l}\text { Solar wind } \\
\text { at 40 AU }\end{array}$ & $\begin{array}{l}\text { Galactic } \\
\text { magnetic } \\
\text { field }\end{array}$ & $\begin{array}{l}\text { Magnetic } \\
\text { field in } \\
\text { clusters of } \\
\text { galaxies }\end{array}$ & $\begin{array}{l}\text { The } \\
\text { Universe }\end{array}$ \\
\hline $\begin{array}{l}\text { Lower } \\
\text { bound on } \\
\lambda, \mathrm{cm}\end{array}$ & $3 \times 10^{10}$ & $3 \times 10^{11}$ & $7.5 \times 10^{11}$ & $2 \times 10^{13}$ & $3 \times 10^{21}$ & $3 \times 10^{23}$ & $2 \times 10^{28}$ \\
\hline $\begin{array}{l}\text { Upper } \\
\text { bound on } \\
m_{p h}, \mathrm{~g}\end{array}$ & $10^{-50}$ & $10^{-51}$ & $4 \times 10^{-52}$ & $1.5 \times 10^{-53}$ & $10^{-61}$ & $10^{-63}$ & $1.5 \times 10^{-68}$ \\
\hline
\end{tabular}

This author believes that, at present, the most reliable limit on the photon mass comes from the spacecraft measurements of the magnetic field and a plasma of the Solar wind. Most notorious among them are the Voyager spacecraft, which have already reached the boundary of the heliosphere. Remarkable agreement has been reached between the observed structure of the magnetic field and theory (standard, zero-photonmass, MHD) that takes into account two basic features of the problem: the radial plasma expansion, and rotation of the Sun. The observed average angle between the magnetic field and the direction to the Sun agrees very well with the spacecraft measurements at the distances from Mercury to Pluto [20]. The field strength and its direction also agrees 
well with the theory. All this makes the solar wind a very convenient object for constraining the photon mass. Assuming the finiteness of $m_{p h}$ and comparing predictions of the appropriately modified MHD equations (3)-(7) with observations, one obtains an upper bound for $m_{p h}$. By choosing large safety margin, one obtains an estimate as reliable as on could obtain in the laboratory. This approach has been implemented in this paper for the Solar wind at large distances from the Sun. The resulting (recommended) estimate is given by Eq. (16).

Further improvements may come from three directions. The first is a more detailed analysis of the Solar wind, with detailed numerical simulations of the flow patterns arising from the finiteness of $m_{p h}$ and comparing the results with observations. This may give rise to the improvement (reduction) of the mass estimate by a factor 5-10.

The second is applying an analysis of the type presented in Sec. 3.2, to the relatively compact objects ( $\sim 1$ pc scale) like dense molecular clouds in HII regions. There already exists a large amount of observational information about magnetic fields and plasma parameters in such bodies, and general understanding of their dynamics gradually emerges.

The third is using the presence of large-scale fields in galaxies and galaxy clusters. This can potentially lead to a dramatic improvement of the mass limit. There are, however, difficulties in making reliable predictions associated with insufficient knowledge of the underlying dynamics. The simultaneous presence of both large-scale field, with length-scale exceeding $\lambda$, and of a stronger, small-scale field with a length scale less than $\lambda$, may make the analysis quite difficult.

A summary of some available estimates of the photon mass is presented in Table 1. The last column in this paper represents a so-called "ultimate" limit on the photon mass, that is imposed simply by the presence of a finite spatial scale of the Universe [4, 10]. One sees that even the best conceivable estimates of the photon mass are still far beyond the ultimate limit. New ideas are needed to bridge this gap.

\section{Acknowledgment}

The author is grateful to E. Synakowski and D.N. Hill for their support and encouragement. This work was performed under the auspices of the U.S. Department of Energy by University of California Lawrence Livermore National Laboratory under contract No. W-7405-Eng-48

\section{References}

[1] A. Proca. "Sur l'equation de Dirac" Compt. Rend. 190, 1377 (1930).

[2] L.D. Landau, E.M. Lifshitz. "Quantum Theory of Fields" (Oxford, Pergamon, 1987).

[3] J.D. Jackson. "Classical Electrodynamics" (Chichester, Wiley, 1975).

[4] A.S. Goldhaber, M.M. Nieto. "Terrestrial and Extraterrestrial Limits on the Photon Mass.” Rev. Mod. Phys., 43, 277 (1971).

[5] J.C. Byrne. "Cosmic tests of Maxwell's equations.” Astrophys. Space Sci., 46, 115 (1977).

[6] A. Zellinger, G. Weihs, T. Jennewein, M. Aspelmeyer. "Happy centenary, photon." Nature, 433, 230 (2005). 
[8] L.C. Tu, J. Luo, G.T. Gilles. "The mass of photon.” Rep. Progr. Phys., 68, 77 (2005).

[9] L.B. Okun. "Photon: history, mass, charge." Acta Physica Polonica, 37, 565 (2006).

[10] S. Eidelman, and Particle Phys. Group. "Review of particle physics," Phys. Lett. B592, 1 (2004); W.-M. Yao and Particle Data Group. "Review of particle physics," J. Phys. G, 33, 1 (2006).

[11] J. Luo, et al. "New experimental limit on the photon rest mass with a rotating torsion balance." Phys. Rev. Lett., 90, 081801 (2003).

[12] A. Goldhaber, M. Nieto. "Problems with the rotating-torsion-balance limit on the photon mass." Phys. Rev. Lett., 91, 149101, (2003).

[13] L. de Broglie. "La mechanique ondulatoire du photon. Une nouvelle theorie de la lumiere." v. 1, p. 39, Paris, 1940.

[14] E. Schroedinger. "The Earth's and the Sun's Permanent Magnetic Fields in the Unitary Field Theory." Proc. Royal Irish Academy, A49, 135 (1943).

[15] L. Davis, A.S. Goldhaber, M.M. Nieto. Limit on the photon mass deduced from Pioneer-10 observations of Jupiter's magnetic field. Phys. Rev. Lett., 35, 1402 (1975).

[16] D.D. Ryutov. "The role of finite photon mass in magnetohydrodynamics of space plasmas." Plasma Phys. Controlled Fusion, 39, A73 (1997).

[17] E. Adelberger, G. Dvali, A. Gruzinov. "Photon mass destroyed by vortices." Phys. Rev. Lett., 98, 010402 (2007).

[18] M. Fullekrug. "Probing the speed of light with radio waves at extremely low frequencies." Phys. Rev. Lett., 93, 043901 (2004).

[19] A.J. Hundhausen. Introduction to Space Physics, Ed. M.G. Kivelson and C.T. Russel, Cambridge University Press, 1995.

[20] Solar Wind Eleven. ESA Conference Proceedings, ESA SP 592, European Space Agency, 2005

[21] Parker, E. N., "Dynamics of the Interplanetary Gas and Magnetic Fields", Astrophys. Journ., 128, 664 (1958).

[22] F. Bagenal et al. "Pluto's interaction with the Solar wind." In: Pluto and Charon, Univ. of Arizona Press, 1997

[23] E. Williams, D. Park. "Photon mass and the galactic magnetic field." Phys. Rev. Lett., 26, 1651 (1971).

[24] Y. Yamaguchi. "A composite theory of elementary particles." Progr. Theor. Phys., \#11, 1 (1959).

[25] G.V. Chibisov. "Astrophysical upper limit on the photon rest mass." Sov. Phys. Uspekhi, 19, 624 (1976).

[26] R.M. Barnett and Particle Data Group. "Review of particle physics," Phys. Rev. D, 54, 1 (1996).

[27] D.D. Ryutov. "The virial theorem for the interstellar medium." Prepared for submittal to Astrophysical Journal, June 2007.

[28] K.M. Ferriere. "The interstellar environment of our galaxy." Rev. Mod. Phys., 73, 1031 (2001).

[29] J.-L. Han. "The Galactic magnetic field." Journ. of Phys., Conf. Series, 47, 120 (2006).

[30] R. Beck, A. Shukurov, D. Sokoloff, R. Wielebinski. "Systematic bias in interstellar magnetic field estimates." Astronomy \& Astrophysics, 411, 99 (2003).

[31] C.L. Carili, G.B. Taylor. Ann. Rev. Astron. Astrophys., 40, 319 (2002). 
[32] H. Ohno, M. Takada, K. Dolag, M. Bartelman, N. Sugiyama. "Probing intracluster magnetic fields with cosmic microwave background." Astrophys. Journ., 584, 599 (2003).

[33] T.E. Clarke, P.P. Kronberg, H. Boehringer. "A new radio-x-ray probe of galaxy cluster magnetic field." Astrophys. Journ., 547, L111 (2001).

[34] R.M. Crutcher. "Observations of magnetic fields in molecular clouds." Proc. Conf. "The Magnetized Interstellar Medium," Antalya, Turkey, Sept. 8-12, 2003. Eds. Uyaniker, W. Reich, and R. Wielebinski.

[35] C.F. McKee, E.G. Zweibel. Astrophys. J., 399, 551 (1991).

[36] F. Bertoldi. Astrophys. Journ., 346, 735 (1989); F. Bertoldi, C.F. McKee. Astrophys. Journ., 354, 529 (1990).

[37] D.D. Ryutov, J.O. Kane, A. Mizuta, M.W. Pound, B.A. Remington. "Two models of magnetic support for photoevaporated molecular clouds", Astrophys. Space Sci., 298, 183 (2005).

[38] M. Morris, K. Uchida, T. Do. Nature, 440, 308 (2006).

[39] D.D. Ryutov. "Using astrophysical jets for establishing an upper limit for the photon mass." Bull. Am. Phys. Soc. 49, \#8, 144, (2004).

[40] S.V. Lebedev, A. Ciardi, D.J. Ampleford, S.N. Bland, et al. "Magnetic tower outflows from a radial wire array Z-pinch." Monthly Notices of the Royal Astronomical Society, 361, 97 (2005). 


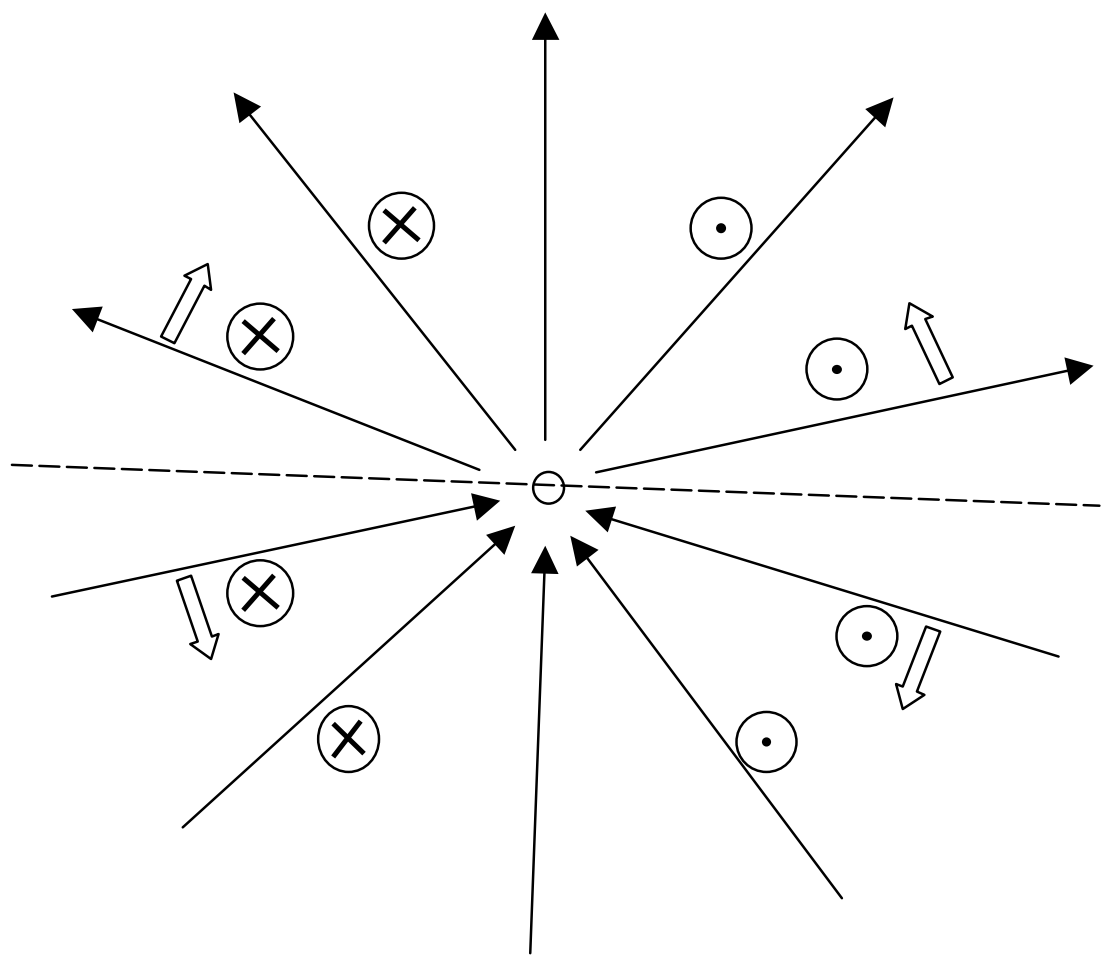

Fig. 1. Schematic of the magnetic field and current distribution inside the Earth orbit. Dashed line represents the equatorial plane. Magnetic field lines are directed radially, with the field direction changing polarity between North and South. Dots and crosses show the direction of the azimuthal plasma current for the distances exceeding the photon Compton length (see Eq. (10)). Block arrows show the direction of the jXB force.
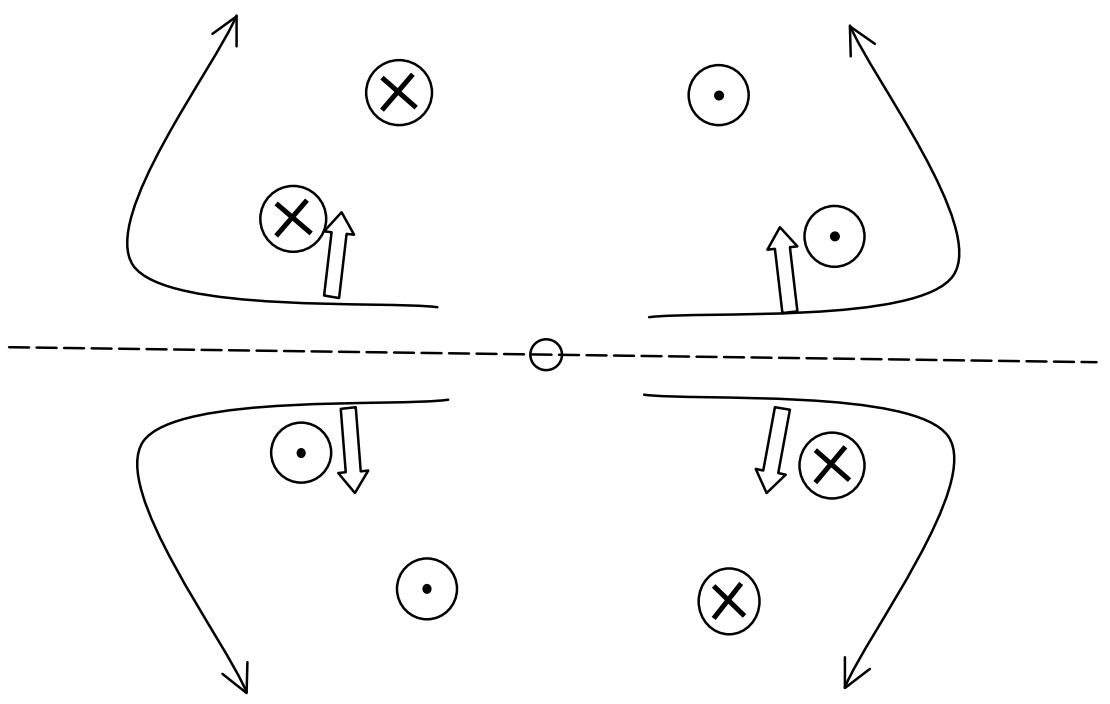

Fig. 2. Schematic of the magnetic field and current distribution at large distances from the Sun (the scale is by a factor of 50 greater than in Fig. 1). The magnetic field is almost purely toroidal, with direction indicated by dots and crosses. Dashed line represents the equatorial plane. Thin lines with arrows show parts of current streamlines. Block arrows show the direction of the $\mathrm{jXB}$ force. 